\title{
0906 ALCOHOL USE AND VIOLENT INJURY IN UGANDA
}

B Wandera*, M Nakiito, R Lett Correspondence: Injury Control Center-Uganda, P.O Box 7072, Kampala Makerere Medical school, Old Mulago Hill, Kampala, Uganda

10.1136/ip.2010.029215.906

Background Alcohol consumption is associated with increased risk of injury. Uganda has the highest alcohol consumption per capita. There is paucity of information about alcohol consumption and injury in Uganda. Moreover this information is relevant in designing appropriate injury prevention interventions. 
Objective To compare injuries by pre-injury alcohol consumption history.

Methods Data were collected between January and December 2009 at emergency departments of five regional referral hospitals from patients presenting with injuries. Analysis for alcohol use restricted to subjects aged above 15 years.

Results Of 3572 injury patients, 2456 patients were above 15 years of whom $307(12.5 \%)$ reported alcohol use before the injury. Persons reporting alcohol use were more likely to be male, $15 \%$ versus $6 \%, p<0.0001$, to have intentional injuries 14.4 versus $6.1 \%, p<0.0001$, seen in city hospital $12.7 \%$ versus $8 \%$, more likely to be brought by police to hospital $18.6 \%$ versus $8.3 \%$, with concussion/coma, $19.5 \%$ versus $10.8 \%$ and more likely to be admitted, $15.5 \%$ versus $10.5 \% \mathrm{p}=0.0002$ but less likely to be involved in a road traffic injury $(10.5 \%$ vs $14.5 \% \mathrm{p}=0.002) .80 \%$ of the intentional injuries were due to interpersonal violence. There were no differences by age nor number of serious injuries sustained.

Conclusions Alcohol use was associated with intentional injuries and is a contributing factor in severe intentional injuries among males in urban areas. Counselling, education and regulation of alcohol consumption needs strengthening. 